Paletschek, Sylvia / Nina Reusch,

Populäre Geschichte und Geschlecht: Einleitung, in: Cheauré, Elisabeth / Sylvia Paletschek / Nina Reusch (Hg.), Geschlecht und Geschichte in populären Medien, Bielefeld 2013, S. 7-37. 


\section{Populäre Geschichte und Geschlecht: Einleitung}

SylVIA PALETSCHEK UND NINA REUSCH

\section{Geschlecht - Geschichte - Historiographie}

Erinnerung und Geschichtsschreibung, egal ob populär oder akademisch, sind immer geschlechtlich kodiert. ${ }^{1}$ Die Kategorie Geschlecht ist sowohl für die Subjekte als auch für die Objekte der Geschichte konstitutiv - wirkt sich also einerseits darauf aus, wer erinnert oder Geschichte schreibt, andererseits darauf, wer und was zum Gegenstand von Geschichtsdarstellungen erhoben wird. Geschlechterdifferenzen strukturieren auch die Institutionen, innerhalb derer Geschichte betrieben wird, die Art historiographischen Arbeitens sowie die Rezeption von Geschichte.

Geschlecht ist somit eine Kategorie, die das gesamte Feld der Geschichtsschreibung und -darstellung durchdringt. Ein gendersensibler Blick auf Geschichtspräsentationen fokussiert nicht allein auf das Geschlecht(erverhältnis) der ProduzentInnen und RezipientInnen, sondern auch auf geschlechtliche $\mathrm{Zu}$ schreibungen sowie Ausschlüsse durch Themenwahl und Perspektive, Medien, Institutionen und Praxen. Intersektional verschränkt mit anderen Ungleichheitskategorien wie z.B. Klasse, Rasse, ethnische und regionale Herkunft, Religion, Alter, körperliche Verfassung etc. bestimmt die Kategorie Geschlecht, wer oder was als geschichtswürdig gilt. Sie konstituiert und durchdringt Wissensräume und Wissensformen.

Einen methodischen Ansatz zur geschlechtersensiblen Untersuchung von Wissen schlägt Monika Mommertz mit dem Konzept vor, Geschlecht als »Markierung, Ressource und Tracer« zu erfassen (Mommertz 2010). Die Kategorie

1 Für die redaktionelle Unterstützung und das Layout dieses Bands danken wir ganz herzlich Josefine Polz. 
,

Geschlecht gibt in dieser Konzeption nicht nur über konkrete Geschlechterverhältnisse Auskunft, sondern auch über diverse gesellschaftliche Bereiche. So lässt sich mithilfe des Konzepts der >Markierung < die Vergeschlechtlichung von (Wissens-)Kulturen analysieren. Dabei können nicht nur Personen geschlechtlich markiert sein, sondern auch Praktiken, Diskurse oder Institutionen. Geschlecht als $>$ Ressource $<$ fragt danach, was die Geschlechterdifferenz beziehungsweise die Zwei- (oder womöglich auch Mehr-)Geschlechtlichkeit für Wissenskulturen und die Produktion von Wissen leistet. Das Konzept des >Tracers < schließlich nutzt die Kategorie Geschlecht als Erkenntnismittel zur Erforschung verschiedener historischer Kontexte. So sind z.B. über die Untersuchung geschlechtsspezifischer Markierungen in der Zuschreibung von wissenschaftlicher Exzellenz und Innovation grundlegende Erkenntnisse über Wissen(schaft)sentwicklungen und Wissensordnungen >im Allgemeinen $<$ möglich.

\section{GESCHLECHT UND AKADEMISCHE HISTORIOGRAPHIE: ERBE DES 19. JAHRHUNDERTS}

Die Art, wie akademische und populäre Geschichte auch heute noch betrieben und vergeschlechtlicht wird, hat ihre Ursprünge in der bürgerlichen Gesellschaft des 19. Jahrhunderts (Paletschek/Schraut 2008b: 10, 23). Mit der beginnenden Akademisierung und Professionalisierung der Historiographie seit dem ersten Drittel des 19. Jahrhunderts weitete sich die Kluft zwischen populärer und akademischer Geschichtsschreibung. Dieses Auseinandertreten und die Verwissenschaftlichung von Geschichte gingen gleichzeitig einher mit einer geschlechtsspezifischen Arbeitsteilung und Themenwahl im Bereich der Wissensproduktion (Epple/Schaser 2009: 7-26; Smith 2001). Die moderne bürgerliche Gesellschaft verwies Frauen idealtypisch auf einen privaten Wirkungsbereich und schloss sie nicht nur aus der politischen Gemeinschaft der Staatsbürger, sondern weitgehend auch aus der wissenschaftlichen Wissensproduktion aus.

Das Resultat war erstens eine Marginalisierung geschichtsschreibender Frauen, die in einer professionalisierten Historikerzunft bis Ende des 19. Jahrhunderts weder eine wissenschaftliche Ausbildung erhalten noch akademische Titel erwerben konnten. ${ }^{2}$ Frauen wurden ab den 1860er Jahren allmählich an einzel-

2 Als erste deutsche Historikerin erwarb Ricarda Huch 1892 in Zürich mit einer Dissertation über »Die Neutralität der Eidgenossenschaft während des Spanischen Erbfolgekrieges« den Doktorgrad (zu Huch siehe Schaser 2012). 1897 promovierte in Heidelhero die erste Histnrikerin an einer deutschen Universität im Gasthörerinnenstatus. 
nen europäischen Universitäten insbesondere der Schweiz, Frankreichs, Schwedens und Großbritanniens, häufig zunächst nur an Frauencolleges oder beschränkt auf bestimmte Fachgebiete oder Fachkurse wie z.B. Medizin an der Petersburger Universität, zum Studium zugelassen. Die Zulassung ging nicht immer mit dem Recht einher, auch einen Abschluss zu machen. Seit den 1880er Jahren öffneten sich sukzessive die Universitäten einzelner Staaten für das Frauenstudium, so in Frankreich, Norwegen, Schweden, Belgien oder Italien. Um die Jahrhundertwende folgten die Habsburger Monarchie, Russland und schließlich auch die deutschen Universitäten (Schneider 2006: 4-5). Erstmals konnten Frauen 1900 an badischen Universitäten als ordentliche Studentinnen zugelassen werden; bis 1909 öffneten schließlich alle deutschen Einzelstaaten ihre Universitäten für Frauen. Seit den 1890er Jahren kam es zu ersten geschichtswissenschaftlichen Promotionen von Frauen, vor allem von amerikanischen und englischen, aber auch deutschen Historikerinnen. Doch nicht nur der Zugang zu universitärer Ausbildung und Abschlüssen, sondern auch zu den historischen Wissensbeständen in Universitätsbibliotheken und Archiven war für Frauen lange noch beschränkt; ihnen blieben also die meisten infrastrukturellen und institutionellen Voraussetzungen der akademisch-historiographischen Forschung verwehrt (Epple 2011: 27; Grever 1997: 116).

Am Beispiel des Frauenstudiums lässt sich das Konzept von Geschlecht als Marker, Ressource und Tracer gut darstellen: Die Nichtzulassung von Frauen an deutschen Universitäten im 19. Jahrhundert - der formale Grund war das fehlende Abitur - markierte Studium und Universität als männlichen Raum. Die geschlechtsspezifische Arbeitsteilung stellte eine wichtige Ressource für die akademische Wissensproduktion dar, entlastete sie doch den Wissenschaftler von Reproduktionsaufgaben und erlaubte es ihm, sich ganz seiner Arbeit zu widmen. Der Blick auf die geschlechtsspezifische Dimension wissenschaftlicher Produktion und die Suche nach dem Beitrag von Frauen zur Wissenschaft macht - und hier fungiert Geschlecht als Tracer - die doppelte >Schattenarbeit « im Hintergrund der oft als geniale Einzelleistung des männlichen Wissenschaftlers dargestellten wissenschaftlichen Arbeit deutlich: Nicht nur war die Freistellung von häuslichen und familiären Arbeiten eine Voraussetzung dafür, sondern ebenso wissenschaftliche Zuarbeiten in Form von Recherchieren, Redigieren, Übersetzen, Messen, Verzeichnen etc. Diese - keineswegs immer nur von Frauen übernommenen - Tätigkeiten werden bei der >Suche< nach Frauen in der Wissen- 
schaft plötzlich sichtbar und lassen so die komplexen, für die Produktion wissenschaftlichen Wissens notwendigen Bedingungen aufscheinen. Frauen waren daher als Privatassistentinnen, als Ehefrauen, Schwestern oder Töchter von akademischen Historikern auch vor ihrer offiziellen Zulassung zum Universitätsstudium und zur Promotion durchaus am Prozess der akademischen historischen Wissensgenerierung beteiligt (Paletschek 2007: 109-111).

Formal jedoch aus dem akademischen Wissensraum ausgeschlossen, blieben geschichtsschreibende Frauen verstärkt auf den Bereich der spopulären< Geschichte verwiesen (Grever 1997: 2009). Doch war auch dieses historische Feld gegendert: zwar finden wir im 19. Jahrhundert erfolgreiche historische >Sachbuchautorinnen< wie etwa Fanny Arndt, doch war ihr Anteil unter den Autoren populärer Geschichtsschreibung marginal (Nissen 2009: 74-77). Auch wählten sie, ähnlich wie ihre männlichen Kollegen, hauptsächlich Themen der Politikgeschichte oder die Biographien >großer Männer< als Gegenstand. Dagegen waren Autorinnen im Bereich des historischen Romans, d.h. der fiktionalen Geschichtsschreibung, stärker vertreten. Dies hatte vermutlich sowohl ökonomische Gründe - vom Verkauf historischer Romane ließ sich eher leben als von Sachbüchern - als auch strukturelle Ursachen. Der faktuale Wissenserwerb war für Frauen aufgrund der Rollenvorgaben, des Bildungssystems und der rechtlichen Beschränkungen schwieriger; und ihnen wurde die Deutungshoheit in Sachfragen der historischen Wirklichkeit nicht im selben Maße wie Männern zugesprochen und zugetraut.

Zum zweiten wandte sich die akademische Geschichtsschreibung des 19. Jahrhunderts primär bürgerlich-männlichen Lebensräumen und damit verbundenen Themen wie Staat, Kirche, Nation oder Krieg zu. Zwar finden wir zum Teil in der Aufklärungshistorie des späten 18. Jahrhunderts noch etablierte Historiker, die sich mit Frauengeschichte beschäftigten (Meiners 1788-1800; StollbergRilinger 1996), im Verlaufe des 19. Jahrhunderts waren es aber vornehmlich Außenseiter der Zunft, wie etwa der Kulturhistoriker Johannes Scherr, die sich der Geschichte von Frauen widmeten (Scherr 1860). Der mainstream der akademischen Historiker behandelte vornehmlich Themen und Institutionen der politischen Geschichte sowie die Ideen, die Werke und das Handeln >großer Männer<. Diese Sujets wurden als geschichtswürdig begriffen und ihre geschlechtliche Markierung - die Themen der Historiographie drehten sich allesamt um männliche Handlungsräume - wurde verdeckt bzw. als allgemeinhistorisch gesetzt (Epple/Schaser 2009; Paletschek/Schraut 2008c: 268). Gleichzeitig sah man mit der Konzentration auf Politik- und Ideengeschichte historische Themen, die weiblich konnotiert oder mit weiblichen Lebensbereichen verbunden waren - so kultur- und sozialgeschichtliche Themen wie z.B. Ernährung, Körper, Geburt 
und Tod, Familie, Alltagsleben etc. - als weniger bedeutsam an, oder sie waren gar kein Gegenstand der wissenschaftlichen, männlich kodierten Geschichtsschreibung.

Weibliche Lebenswelten wurden nicht nur in der akademischen Geschichtsschreibung, sondern auch in der historischen Erinnerungskultur marginalisiert, indem sie im Bereich anthropologischer Konstanten verortet und damit enthistorisiert wurden. Frauen erinnerte man in ihrer Funktion als Mütter; weibliche Symbole standen für überzeitlich gedachte Werte, so etwa die Nation. Mit Geburt und Lebenskreislauf, mit Emotionen und der Natur assoziiert, repräsentierten Frauen bzw. weibliche Symbole ein zyklisches Zeitmodell, das als ahistorisch, d.h. unveränderlich erschien. Die Geschichte hingegen, verstanden als permanente historische Veränderung und als (teleologisches) Fortschreiten, wurde männlich gédacht (Paletschek/Schraut 2008b: 26).

Die Marginalisierung von Frauen war also eine doppelte - sowohl als Subjekte, d.h. als Geschichtsschreiberinnen, als auch als Objekte, d.h. potentielle Akteurinnen der Geschichte (Smith 1984; Smith 2001: 103-156). Populäre Formen der Geschichte bargen jedoch schon im 19. Jahrhundert die Möglichkeit, die männliche Markierung von Geschichte zumindest teilweise aufzubrechen. Wie Bonnie Smith und Angelika Epple (Smith 2001: 37-69; Epple 2003) gezeigt haben, gab es in den Jahrzehnten um 1800 auf dem Buchmarkt erfolgreiche Historikerinnen wie z.B. Catherine Macauly, die Strickland Schwestern, Therese Huber oder Johanna Schopenhauer, die allerdings in der Folgezeit von der auf die akademische Geschichtsschreibung konzentrierten Historiographiegeschichte vergessen wurden. Im literarischen Genre des Romans bearbeiteten zahlreiche Autorinnen historische Stoffe. Historische Romane und andere, nicht an den akademischen Kanon gebundene Formen der Geschichte erlaubten es zudem, Themen zu setzen, die Frauen als historische Akteurinnen ins Licht rückten.

Vor allem Kultur- und Alltagsgeschichte sowie biographische Zugänge hatten das Potential, den auf Staat, Politik, Kirche und große Ideen ausgerichteten Kanon aufzubrechen, und Frauen, aber auch nicht der bürgerliche oder adeligen Elite zugehörige Männer in ihren historischen Lebensweisen und Wirkungsbereichen darzustellen. Gleichzeitig war die Fiktionalisierung historischer Stoffe ein Weg, Frauen sowie untere soziale Schichten auch dann in der Geschichte sichtbar zu machen, wenn keine oder nur wenig Quellen verfügbar waren. Die Frauen- und Geschlechtergeschichte musste und muss sich häufig dem Problem stellen, dass der Alltag von Frauen gerade unterer sozialer Schichten nur selten dokumentiert und überliefert wurde, dass Frauen selbst aufgrund mangelnder Schreibfähigkeit keine schriftlichen Quellen hinterließen oder dass Quellen, die Auskunft über Leben und Alltag von Frauen geben könnten, nicht in dem Maße 
1

archiviert werden wie Quellen, die Auskunft über männlich markierte Personen und Themen geben. Eine Wendung in fiktionale Genres wie den historischen Roman ermöglicht hier, den Mangel an Quellen zu kompensieren und auch auf lückenhafter Quellenbasis Frauen- und Alltagsgeschichte zu schreiben.

\section{Frauenbewegungen UND GeSchichtSSCHREIBUNG}

Frauengeschichte wurde im 19. Jahrhundert aber nicht allein in der populären Geschichtsschreibung und von Außenseitern der Zunft, sondern auch von Autorinnen aufgegriffen, die in der Frauenbewegung aktiv waren. Für die seit der Mitte des 19. Jahrhunderts in Europa entstehenden, in den Jahrzehnten um 1900 zu öffentlichkeitswirksamen Massenbewegungen herangewachsenen europäischen Frauenbewegungen war die Beschäftigung mit Geschichte ein wichtiges Moment der Identitätsstiftung und der Legitimation ihrer Forderungen - und zwar auf mehreren Ebenen (Grever 1997; Paletschek/Pietrow-Ennker 2004: 301-307).

Erstens dienten >große Frauen $<$ der Vergangenheit oder der Mythologie zur Legitimation der Emanzipationsforderungen der jeweiligen Gegenwart: so konstruierte etwa die englische Frauenrechtlerin Charlotte Carmichael eine Traditionslinie weiblichen Widerstandsgeistes vom Kampf der Königin Boadicea gegen die Römer bis zu den Frauenrechtsforderungen ihrer Gegenwart (Rendall 2004: 33). Für die frühe tschechische Frauenbewegung waren historische und mythologische Figuren wie die Prinzessin Libuse, die tschechischen Amazonen, die Heilige Ludmila oder die weiblichen Hussiten ein wichtiger Teil ihrer Argumentationsstrategien (Malečkova 2004: 167). Gerade für Frauenbewegungen, die eng mit nationalen Unabhängigkeitsbewegungen verbunden waren, hatte das Einschreiben von Freiheitskämpferinnen oder auch weiblichen Heiligen in die Geschichte der Frauenbewegung wie in die Nationalgeschichte bzw. nationale Mythologie eine wichtige Funktion.

Zweitens waren Aktivistinnen der >alten $<$ Frauenbewegung meist die ersten, die im frühen 20. Jahrhundert eine Geschichte ihrer Bewegung schrieben oder die Initiative ergriffen, Quellen zu ihrer Bewegung zu sammeln. So verfassten Helene Lange und Getrud Bäumer 1901 einen ersten Überblick zur Geschichte der deutschen Frauenbewegung, Johanna Naber unternahm dies für die Niederlande 1908, zur englischen Frauenbewegung erschien 1928 ein großes Werk von Ray Strachey. Seit Ende des 19. Jahrhunderts wurden von den feministischen Aktivistinnen und Aktivisten Archive angelegt. So begannen die holländische Ärztin und Feministin Aletta Jacobs und ihr Mann C.V. Gerritsen, Bücher, 
Pamphlete und vor allem Zeitschriften zur feministischen Bewegung in Europa und Übersee zu sammeln; ${ }^{3}$ gleiches geschah in Frankreich, wobei die von Eliska Vincent seit den 1890er Jahren angelegte Sammlung verloren ging und überhaupt viele dieser Initiativen, so auch das 1935 in Amsterdam gegründete International Archive for the Women's Movement durch den Zweiten Weltkrieg, die deutsche Besatzungszeit und die NS-Verfolgungen zum Stillstand kamen oder vernichtet wurden (Offen 2000: 3-17).

Drittens: Frauenrechtlerinnen der ersten Stunden beschäftigten sich nicht nur mit der engeren >eigene Geschichte< ihrer politischen Bewegung, sondern verfassten allgemeinere Werke zur Geschichte der Frauen oder einzelner herausragender Frauen, so etwa die deutsche Anna Blos zu den Frauen der Revolution von 1848/49 (Blos 1928; Riepl-Schmidt 1998: 134-156) oder die Russin Anna Engel'gardt mit ihrer breit angelegten historischen Arbeit über »Frauen in der Gesellschaft und der Familie« (Pietrow-Ennker 1999: 258).

Die Beispiele zeigen, dass es hier eine vornehmlich von Aktivistinnen verfasste Frauengeschichte schon vor der in den 1970er Jahren aufkommenden akademischen Frauen- und Geschlechtergeschichte gab. Die Beschäftigung mit Frauengeschichte im 19. und 20. Jahrhundert war - wenn auch nicht ausschließlich - insgesamt jedoch sehr eng mit der Entstehung feministischer Bewegungen verknüpft und scheint ohne diese kaum möglich gewesen zu sein. Wie schon die Feministinnen des 19. Jahrhunderts arbeiteten auch die Aktivistinnen der Zweiten Welle der Frauenbewegung seit den 1970er Jahren daran, die Lebenswelten von Frauen in der Geschichte zu erforschen und sie in den historischen Kanon einzuschreiben. Die Frauengeschichte der 1970er und 1980er Jahre erhielt ihren Anstoß aus der sich formierenden zeitgenössischen Frauenbewegung und nicht aus den Universitäten heraus, selbst wenn es häufig feministische Studentinnen und Doktorandinnen bzw. promovierte Historikerinnen waren, die gemeinsam mit anderen Feministinnen in Frauengruppen und Geschichtswerkstätten die Geschichte von Frauen systematisch zu erforschen begannen (zu Frauenarchiven und -bibliotheken vgl. Schatzberg 1985).

Mit der zunehmenden Professionalisierung und Verwissenschaftlichung der Frauengeschichte, d.h. der Weiterentwicklung zur Geschlechtergeschichte und der theoretischen Reflexion der historischen Kategorie Geschlecht seit den 1980er Jahren (Bock 1988; Scott 2003), vollzog sich ein methodischtheoretischer ebenso wie personell-institutioneller Abgrenzungsprozess der aka-

3 Heute ist diese Bibliothek bzw. dieses Archiv online zugänglich und stellt einen wichtigen Quellenfundus für die Geschichte der europäischen Frauenbewegungen dar (http://gerritsen.chadwyck.com/home.do). 
demischen Frauen- und Geschlechtergeschichte von einer außeruniversitären, identitär und frauenpolitisch fokussierten >Bewegungsgeschichtsschreibung< (Frevert 1988: 240-262; zu feministischer Identitätspolitik vgl. Maurer 1996; Hunt 1998). Die akademische Anerkennung der Frauen- und Geschlechtergeschichte, ebenso wie der Einstieg der zu diesem Themenfeld arbeitenden Historikerinnen (und sehr wenigen männlichen Historiker) in Universitätskarrieren ging mit einer Distanzierung von den politischen Frauenbewegungen einher (Scott 2004: 10-29; Bennett 2006; Paletschek 2009: 172). Der neuen Geschlechtergeschichte wurde deshalb der Vorwurf gemacht, politisch sytemstabilisierend und quietistisch zu wirken und das subversive politische Potential der Frauengeschichte auszuhebeln (Hunt 1998: 57-98). Wie Krista Cowman am britischen Beispiel zeigen konnte, wurde die von Laien-Historikerinnen und feministischen Aktivistinnen geschriebene Frauengeschichte von der neuen, universitären Frauen- und Geschlechterhistoriographie marginalisiert oder ausgeblendet; damit wurde auch, bei aller nachvollziehbaren Kritik an dieser oft intentional, auf politische Legitimation hin angelegten >populären< feministischen Geschichtsschreibung, deren partiell vorhandenes innovatives Potential negiert bzw. übersehen (Cowman 2009). Sowohl die akademische Frauen- und Geschlechterhistoriographie als auch ihr >populärer < feministischer Gegenpart hatten, wie das Beispiel zeigt, blinde Flecken. Sie konzentrierten sich auf Themen, die für den jeweiligen Rezeptionskontext und seine Denkstile bedeutsam waren, und nahmen sich gegenseitig nur mehr partiell wahr.

\section{FRAUEN- UND GESCHLECHTERGESCHICHTE, WISSENSCHAFTSBETRIEB UND HISTORISCHER KANON}

Die wissenschaftliche Etablierung der Frauen- und Geschlechtergeschichte - wie der Genderforschung insgesamt - hat einerseits während der letzten drei Jahrzehnte beachtliche Erfolge zu verzeichnen: eine Vielzahl von Forschungsarbeiten entstand, Professuren mit einer Denomination bzw. Teildenomination für Frauen- und Geschlechtergeschichte wurden errichtet, Fachzeitschriften ${ }^{4}$ sowie wissenschaftliche Fachorganisationen wie der »Arbeitskreis für Frauen- und Geschlechtergeschichte (AKHFG)« (2007) bzw. international die »International Federation of Research in Women's History (IFRWH)« (1987) gegründet. Ande-

4. Feministische Studien (1982); Ariadne: Forum für Frauen- und Geschlechtergeschichte (1985); Journal of Women's History (1989); L'homme: Europäische Zeitschrift für feminictierhe Fecrhirhtswissenschaft (1990): Gender \& Historv (2004). 
rerseits ist das Einschreiben der Analysekategorie Geschlecht in den historischen Kanon bis heute nicht vollständig gelungen (Hagemann/Quataert 2008: 27-47). Ergebnisse der Frauen- und Geschlechtergeschichte werden auch heute meist nur additiv der großen (faktisch noch immer um männliche Lebensräume zentrierten) historischen Erzählung beigefügt. Eine symmetrische Geschichtsschreibung mit einer gendersensible Perspektive als integralem Bestandteil (vgl. zu den Forderungen Scott 2003; Bock 1988; Medick/Trepp 1998; Hausen 1998) ist weiterhin Desiderat, insbesondere was übergreifende synthetische Geschichtsdarstellungen in Überblickswerken oder Handbüchern zu National- oder neuerdings Globalgeschichte angeht (Smith 2009: 33-41). Eine Möglichkeit, männliche Kodierungen von Geschichte zu dekonstruieren, wird z.B. darin gesehen, multiple Geschichten in vielfältigen räumlichen Bezügen auch außerhalb der nationalen Kanons in die historiographische Forschung einzubeziehen (Epple 2009; Grever 2009: 53-54). Die trotz der zu konstatierenden Erfolge der Frauen- und Geschlechtergeschichte oftmals fehlende Sensibilität für die Differenzkategorie Geschlecht ist auf die große Beharrungskraft wissenschaftlicher Traditionen und Relevanzzuschreibungen, auf die noch deutlich optimierbare institutionelle Verankerung der Frauen- und Geschlechtergeschichte an den Universitäten, ebenso wie auf die institutionelle Geschlechterungleichheit an den Universitäten ${ }^{5}$ sowie in gesellschaftlichen Führungspositionen zurückzuführen.

Warum es so schwierig ist, einen geschlechtersensiblen Perspektivwechsel im historischen Kanon und Geschichtsverständnis zu verankern, hat auch damit zu tun, wie Geschichtsschreibung organisiert ist und welche Rolle Geschlecht in der Erinnerungs- bzw. Geschichtskultur spielt. Untersuchungen der Historiographiegeschichte und der Erinnerungs- bzw. Geschichtskultur unter geschlechtergeschichtlichen Fragestellungen können hier ein wichtiges tool sein, um nicht nur vergangene, sondern auch gegenwärtig noch wirksame und tradierte Einund Ausschlussmechanismen zu verstehen und dadurch ein Stuick weit auszuhebeln. Bonnie Smith hat in ihren bahnbrechenden Arbeiten über »The Gender of History« (Smith 2001; Smith 1984) die männliche Imprägnierung und Imaginierung der im 19. Jahrhundert sich entwickelnden wissenschaftlichen Geschichts-

5 Frauen stellen heute zwar über die Hälfte der Geschichtsstudierenden, ihr Anteil nimmt jedoch mit jeder wejteren universitären Karrierestufe ab. Der Anteil von Geschichtsprofessorinnen lag 2002 bei ca. 12\% (Paletschek 2007: 124); wie auch in anderen wissenschaftlichen Disziplinen ist auch in der Geschichte seit den 2000er Jahren ein deutlicher Anstieg des Professorinnenanteils zu verzeichnen; allerdings sind die prestigereichen, gut ausgestatteten Professuren überproportional häufig mit Männern besetzt. 
11

schreibung herausgearbeitet und mit als erste den Blick nicht nur auf die universitäre, sondern auch auf die außeruniversitäre und populäre Geschichtsschreibung von Frauen gelenkt. Ebenfalls früh hat auch Maria Grever darauf hingewiesen, dass die Untersuchung von Historikerinnen außerhalb der Akademien nötig sei, um die »relative Geschichtslosigkeit der Frauen« einerseits als Resultat und Teil der Ausschlussmechanismen des wissenschaftlichen Betriebs zu erfassen, andererseits aber auch die Grenzen von populärer, literarischer und akademischer Geschichtsschreibung, die im Zuge der Professionalisierung der Geschichtswissenschaft entstanden, als Genre- wie Geschlechtergrenzen zu verstehen (Grever 1997; vgl. auch Howsam 2004; Maitzen 1998).

In den letzten 15 Jahren entstanden im Anschluss an diese Forschungen Untersuchungen, die sich mit der Metaebene der Historiographiegeschichte unter Genderperspektive beschäftigten - also z.B. mit der Geschichte von Historikerinnen oder der Verhandlung von männlichen Geschlechtsindentitäten in der Geschichtschreibung. ${ }^{6}$ Geschichte schreibende Frauen, das zeigen diese Studien, verrichteten ihre Arbeit bis weit ins 20. Jahrhundert hinein fast ausschließlich im Spannungsfeld von populärer und wissenschaftlicher Historiographie. So war es auch ein Verdienst der Geschlechtergeschichte - gewissermaßen als Tracer populären Geschichtspräsentationen wie auch ihren Interaktionen mit der akademischen Historiographie mehr Aufmerksamkeit zukommen zu lassen und aufzuzeigen, dass es vielfältige Verflechtungen zwischen akademischer und außerakademischer Historiographie gab und gibt.

Die akademische Geschichtsschreibung ist, ebenso wie die populäre, nicht getrennt von der in einer Zeit vorherrschenden Geschichtskultur zu begreifen; beide sind in je spezifischer Weise von dieser beeinflusst. Keineswegs kann die wissenschaftliche Geschichtsschreibung, wie noch von Maurice Halbwachs so gesehen, als säuberlich getrennt von der vorherrschenden Erinnerungskultur betrachtet werden (Halbwachs 1985). Sie geht zwar nicht in dieser auf - aufgrund der geforderten wissenschaftlichen Selbstreflexion und des historistischen Codes, jede Zeit aus sich heraus zu verstehen und methodisch kontrolliertes historischen Wissen zu produzieren, ist sie weniger intentional und funktional ausgerichtet als das kollektive Gedächtnis. Doch zeigen gerade die Forschungen zur Nationalgeschichtsschreibung (Berger 2008), aber auch zur männlichbürgerlichen Imprägnierung der modernen Historiographie, dass diese keines-

6 Siehe u.a. Melman (1993); Stollberg-Rillinger (1996); Grever (1997); Maitzen (1998); Medick/Trepp (1998); Burstein (1999); Epple (2003; 2011); O’Dowd (2004); Paletschek (2006; 2007a); Felber (2007); Berger (2008); Hagemann/Quataert (2008); Epp- 
wegs unabhängig von den erinnerungskulturellen und geschichtspolitischen Bedürfnissen ihrer Gegenwart operiert. Thre Verschränktheit mit Erinnerungskulturen, die wiederum von den jeweiligen Gesellschafts- und Geschlechterverhältnissen geprägt sind, verstärkte z.B. im 19. und auch noch im 20. Jahrhundert die Ausrichtung der wissenschaftlichen Geschichtsschreibung auf männliche Handlungsräume.

\section{ERINNERUNGSKULTUR, GESCHICHTSKULTUR, POPULÄRE GESCHICHTE UND GESCHLECHT}

Mittlerweile kursieren eine Vielzahl von Begriffen und Konzepten, die sich im weitesten Sinne auf den Umgang einer Gesellschaft mit der Vergangenheit und auf deren Präsentation und Funktion beziehen: kollektives Gedächtnis (Halbwachs), kulturelles und kommunikatives Gedächtnis (J. und A. Assmann), Erinnerungsorte (Nora), Erinnerungskultur (J. und A. Assmann, Gießener SFB, Fran çois/Schulze), Geschichtskultur (Rüsen, Schönemann), Geschichtspolitik (Wolfrum), Erinnerungsgeschichte (Langewiesche), Public History oder populäre Geschichte bzw. Geschichte in populären Medien und Genres (Korte/Paletschek).

Einflussreich war hier zunächst die Theorie des kollektiven Gedächtnisses, die Maurice Halbwachs bereits in den 1920er Jahren formulierte (Halbwachs 1985) und die darauf abhob, dass Erinnerung immer rückgebunden ist an ein Kollektiv, dass nur in einem sozialen Rahmen erinnert werden kann und Erinnerung für die Identität einer Gruppe funktionalisiert wird. Seit Ende der 1980er Jahre wurde diese Theorie von Aleida und Jan Assmann aufgenommen und zum Modell des kulturellen und kommunikativen Gedächtnisses bzw. des Speicherund Funktionsgedächtnisses ausgearbeitet (Assmann 1992; Assmann/Assmann 1994). Kulturelles Gedächtnis fasst den in

»jeder Gesellschaft oder jeder Epoche eigentümlichen Bestand an WiedergebrauchsTexten, -Bildern und -Riten zusammen, in deren >Pflege< sie ihr Selbstbild stabilisiert und vermittelt, ein kollektiv geteiltes Wissen vorzugsweise (aber nicht ausschließlich) über die Vergangenheit, auf das eire Gruppe ihr Bewußtsein von Einheit und Eigenart stützt.« (Assmann 1988: 15)

Erinnerungskultur wird verstanden als »Gedächtnis, das Gemeinschaft « (Assmann 1992: 30) stiftet und hebt besonders auf den funktionalen Gebrauch für 
gegenwärtige (politische) Zwecke und historisch begründete Identitäten ab (Cornelißen 2003: 555). ${ }^{7}$

Nicht nur die Kulturwissenschaften, auch die Geschichtsdidaktik hat sich fast zeitgleich der Frage nach dem Umgang mit Geschichte und ihrer Bedeutung für die Gesellschaft angenommen und das Konzept der Geschichtskultur entwickelt (Rüsen 1994; Schönemann 2002). Geschichtskultur umfasst alle in einer Gesellschaft wirkenden oder aufzufindenden Formen des Geschichts- oder Vergangenheitsbewusstseins. ${ }^{8}$ Geschichtskultur-Forschung untersucht die vielfältigen Arten, wie sich eine Gesellschaft in Beziehung zu ihrer Vergangenheit setzt und berücksichtigt dabei, dass Geschichte nicht nur kognitiv in bewussten Erinnerungsakten, sondern auch ästhetisch und emotional rezipiert wird, dass sie vielfältige Funktionen - der Belehrung wie der Kritik, der Legitimation wie der Aufklärung, aber auch der Unterhaltung und Ablenkung - erfüllen kann. Sie wird nicht nur von staatlichen und kulturellen (Bildungs-)Institutionen, sondern ebenso von kommerziellen Einrichtungen und Medien sowie zivilgesellschaftlichen Vereinigungen getragen. Unter dem umbrella term Geschichtskultur werden nicht nur historische Inhalte und Narrative untersucht, sondern auch die AkteurInnen und Medien, die Geschichte konstruieren, ebenso wie die Verbreitungswege und die Rezeption des historischen Wissens (Grever 2008; Sánchez Marcos 2009). Das Konzept der Geschichtskultur ist weniger prozesshaft-funktional ausgelegt als andere Konzepte des Umgangs mit Geschichte. Es fasst daher den bei populären Geschichtspräsentationen oft in den Vordergrund tretenden ästhetischen und ökonomischen, spielerisch-unterhaltenden und auch eskapistischen Charakter besser als der auf politische Implikationen ausgerichtete Begriff der Erinnerungskultur.

Stärker auf die Produkt- und Erscheinungsebene fokussiert ist der Begriff der populären Geschichtspräsentationen oder der populären Geschichte, unter dem

7. $\mathrm{Zu}$ unterschiedlichen Dimensionen der Erinnerungskultur (materiell, sozial, mental) siehe Erll (2005: 101ff); zur konzisen Diskussion der unterschiedlichen Konzepte in ihrem Potential zur Analyse von Geschichtsdarstellungen in populären Medien siehe Heinze (2012: 23-35).

8 Der Begriff Vergangenheitsbewusstsein wird aus ethnologischer Perspektive z.B. von Judith Schlehe favorisiert, da der Geschichtsbegriff eine seit der Sattelzeit um 1800 in Europa formierte Vorstellung von Geschichte als Kollektivsingular und fortschreitendem Prozess benennt, die so in vielen nicht-europäischen Gesellschaften nicht ausgeprägt war. Stattdessen waren außerhalb der westlichen Welt häufig religiöse oder mythische Vergangenheitsbezüge konstitutiv, die vielfach noch bis in die Gegenwart weiterwirken. 
Darstellungen in textueller, visueller, audiovisueller und performativer Form gefasst werden, die »Wissen über die historische Vergangenheit in einer verständlichen, attraktiven Weise präsentieren und ein breites Publikum erreichen, das aber nicht unbedingt ein Massenpublikum sein muss.« (Korte/Paletschek 2009b: 13). ${ }^{9}$ Mithilfe des Konzepts populärer Geschichte wird versucht, die breite Palette und die vielfältigen Erscheinungsformen vergangener und gegenwärtiger Beschäftigung mit Vergangenheit zu fassen. Heute können wir Geschichte im Fernsehen und Radio, in Zeitungen, Romanen oder Comics konsumieren. Sie ist präsent in Themenparks und Freilichtmuseen, alten Burgen, Stadtmauern oder Gedenkstätten. Laiendarsteller spielen in Re-Enactments historische Schlachten nach oder schlüpfen auf Mittelaltermärkten und Live-Rollenspielen in historische Lebenswelten. In Computerspielen können UserInnen in historischen Welten agieren, und das Internet bietet zahlreiche Möglichkeiten textueller wie audiovisueller Geschichtspopularisierung. Geschichte war auch schon im 18. und 19. Jahrhundert - und teilweise auch davor - Thema populärer Unterhaltung. Ob es sich nun um historische Bezüge in den Dramen Shakespeares handelt oder um Panoramen des 19. Jahrhunderts, um Bildungszeitschriften der Aufklärung oder um Nationaldenkmäler und Heimatmuseen, die im späten 19. Jahrhundert errichtet wurden - ihnen allen ist gemein, dass sie Geschichte schon lang vor bekannten TV-Sendungen wie »Holocaust« (1978) oder »Hitlers Helfer« (1996) popularisierten. Gewandelt haben sich im 20. Jahrhundert allerdings die Medien und Genres der populären Geschichte. Während Geschichte im 19. Jahrhundert vorwiegend in Printmedien und Museen präsent war oder BesucherInnen von Bauwerken, Ruinen und Denkmälern eine historische Aura vor Ort erleben konnten, bot die Entwicklung vor allem audiovisueller Medien im 20. Jahrhundert völlig neue Möglichkeiten, Geschichte in bewegten Bildern und Tönen lebendig werden zu lassen.

Diè Fokussierung populärer Geschichtspräsentationen auf der Produktebene, aber auch die Frage nach den gesellschaftlichen und individuellen Funktionen von (populärer) Geschichte prädestiniert sie für einen interdisziplinären Zugriff. So legt die Analyse der jeweiligen Mediendispositive, die die historischen Inhalte und das historisch Sag- und Darstellbare bestimmen, sowohl historische, ethnologische wie auch literatur-, kultur- und medienwissenschaftliche Zugänge sowie selbstverständlich auch eine Gender-Perspektive nahe.

Während lange Zeit Facetten der Geschichts- und Erinnerungskultur vornehmlich in ihrer nationalpolitischen Dimension, über >hochkulturelleく Ge-

9 Dort auch weitere Ausführungen zu den Begriffen populär bzw. populäre Geschichtskultur (ebd. 14-20). 
,

schichtsrepräsentationen sowie in Bezug auf Prozesse der Vergangenheitsbewältigung, den Umgang mit Krieg, Traumata und Genozid im 20. Jahrhundert untersucht wurden, erschienen in den letzten Jahren vermehrt auch Studien zur populären Geschichtskultur. Diese Arbeiten analysieren populäre Geschichte, oftmals mit Rückgriff auf neuere, aus den Cultural Studies stammende Theorien, in unterschiedlichsten Epochen, Räumen und Medien. ${ }^{10}$

Doch auch diese neueren Forschungen betrachteten Geschlecht als Faktor populärer Geschichtskultur zunächst höchstens am Rande. Ähnlich wie die Historiographiegeschichte lange Zeit geschlechterblind betrieben wurde, entstanden trotz des Booms der Erinnerungskulturforschung in den letzten beiden Jahrzehnten erst allmählich Arbeiten zum Themenkomplex Geschlecht und Erinnerungskultur (Paletschek/Schraut 2008a). Diese beschäftigten sich vor allem mit in nationale Erinnerungskulturen eingeschriebenen Geschlechterbildern, etwa am Beispiel der napoleonischen Kriege (Schilling 1998; de Bruyn 2002; Förster 2004; 2012), der Nationaldenkmäler des 19. Jahrhunderts (Rausch 2008: 31-60) oder geschlechtlicher Nationalsymbolik (Falkenhausen 1997). Mittlerweile liegen auch einige Arbeiten zu geschlechtsspezifischen Aspekten der Erinnerung an den Holocaust vor (Eschebach/Wenk 2002; Bruns 2012). Doch eine breite Untersuchung populärer Geschichtskultur unter Geschlechterperspektive ist nach wie vor Desiderat.

Im Bereich populärhistorischer Produkte gibt es zahlreiche Beispiele, die zeigen, dass und wie durch populäre Geschichte in faktualen wie fiktionalen, textuellen, audiovisuellen wie performativen Formen Geschlechterrollen und -verhältnisse in Vergangenheit und Gegenwart verhandelt wurden und werden. So steht etwa bei Mittelaltermärkten oder Freilichtmuseen eine Rekonstruktion historischer Alltagskultur und weiblicher wie männlicher Handlungsräume im Vordergrund; Spielfilme beschäftigen sich mit großen Frauen der Geschichte (Elizabeth, 1998/2007) oder mit Frauen, die aus ihrer traditionellen Geschlechterrolle ausbrechen (The Messenger: The Story of Joan of Arc, 1999; Die Päpstin, 2009); auch Homosexualität rückt zuweilen in den Fokus der historischen

10 Als Auswahl vgl. Rosenzweig/Thelen (1998); Crane (2000); Glassberg (2001); Crivellari et al. (2004); Hardtwig/Schütz (2005); Melman (2006); Groebner (2008); Langewiesche (2008); Bösch/Goschler (2009); Groot (2009); Hardtwig/Schug (2009); Howsam (2009); Korte/Paletschek (2009a; 2012); Nissen (2009); Oswalt (2009); Gehrke/Sénécheau (2010); Pirker/Rüdiger (2010); Korte/Pirker (2011); Paletschek (2011); Samida (2011); Heinze (2012); Kircher (2012); zu Geschichte im Fernsehen siehe u.a. Lersch/Viehoff (2007); Keilbach (2008), Fischer/Wirtz (2008); Horn (onna) 
Populärkultur (Aimée und Jaguar, 1999). In Dokus werden weibliche Rollenmodelle der 1950er Jahre getestet (Die Bräuteschule, 2007; Klein 2009) und in der Re-Enactmentszene ermöglicht die Nachstellung historischer Schlachten die Verwandlung der Teilnehmenden in männliche Krieger und Soldaten. Historische Romane haben selbst in vormodernen Epochen emanzipierte Frauen als Heldinnen (Ken Follett, The Pillars of the Earth, 1990; Iny Lorentz, Die Wanderhure, 2004). In vielen Städten gibt es feministische Stadtführungen bzw: Frauenstadtrundgänge, die vom Touristenbüro vermarktet werden. Werbefilme oder preisgekrönte Fernsehserien wie z.B. »Mad Men« (2007) spielen mit den Geschlechterrollen und -konventionen vergangener Jahrzehnte. Es zeigt sich hier sowohl ein großes Feld für das ständige Wiedereinschreiben hergebrachter Geschlechterrollen wie auch fürs Experimentieren mit geschlechtlichen Identitäten.

Populäre Geschichte vermittelte und vermittelt nicht nur gesellschaftlich relevante Geschichts-, sondern auch Geschlechterbilder, und war und ist ein wichtiger Bestandteil der Konstruktion von Geschlecht und Zweigeschlechtlichkeit. Eine Beschäftigung mit Vergangenheit und Geschichte rekurriert - offen oder verdeckt - immer auch auf die Produktion und Reproduktion von Geschlechterbildern und geschlechtlichen Identitäten, auf die Diskussion, Legitimierung und De-Legitimierung gegenwärtiger Geschlechterverhältnisse sowie auf die historischen Naturalisierungen von Zweigeschlechtlichkeit und bürgerlichen Geschlechter- und Familienmodellen. Durch die Produktion und Rezeption populärer Geschichte findet ein doing gender nicht nur in performativen Bereichen (zum Beispiel durch die spielerische Aneignung historischer vergeschlechtlichter Identitäten) statt. Populäre Geschichte kann auch die Möglichkeit bergen, geschlechtliche Identitäten zu dekonstruieren und zu hinterfragen - so wird (das zeigen einige der Beiträge dieses Bandes) mit Geschlechterrollen vor allem in fiktionalen und performativen Medien gespielt. In der populären Geschichte ist, trotz der affirmativen Dominanz des gesellschaftlichen und geschlechterstereotypen mainstream, zumindest das Potential angelegt, durch Historisierung von Geschlechterverhältnissen das oftmals so selbstverständlich genommene bürgerliche heteronormative Geschlechter- und Familienmodell partiell aufzubrechen und zu dekonstruieren. 
11

\section{Die BeITRÄGE DES BANDES}

Der vorliegende Band analysiert in exemplarischen Fallbeispielen die Zusammenhänge von populärer Geschichte und Geschlecht. ${ }^{11}$ Für historische Referenzräume von der Antike bis zur Gegenwart, für verschiedene Gegenwarten vom 19. bis zum frühen 21. Jahrhundert, sowie für unterschiedliche Medien und Genres wird untersucht, wie Geschlecht und Geschlechterverhältnisse in populären Präsentationen von Geschichte konstruiert und verhandelt werden.

Über die Beschäftigung mit populärer Geschichte kann die Bedeutung der Kategorie Geschlecht für die historische Wissensgenerierung und historische Wissenskulturen reflektiert werden. Mit dem Blick auf die Kategorie Geschlecht als Marker, Ressource und Tracer lassen sich aber auch umgekehrt Spezifika populärer Geschichtspräsentationen differenzierter herauspräparieren. Folgende Forschungsfragen bieten sich als Einstieg an: 1) Welchen Stellenwert haben Themen der Frauen- und Geschlechtergeschichte in populären Medien und welche Entwicklungslinien bzw. Traditionen lassen sich festhalten? 2) Sind populäre Medien, Formate und Publika offener für Fragen der Frauen- und Geschlechtergeschichte als akademische Präsentationsformen und Kontexte? Gibt es eine Interaktion zwischen akademischer und populärer historischer Wissensproduktion in diesem Themenfeld? 3) Wie wirken unterschiedliche Medien und Formate auf die in populären Medien konstruierten Geschichtsbilder von Geschlechterrollen und -verhältnissen zurück? 4) Wie verhalten sich die in populären Medien hergestellten und transportierten Geschichtsbilder zu den jeweiligen Gesellschaftsentwicklungen und welche Rolle spielen populäre Geschichtsdarstellungen in der Herstellung von Geschlecht? Diese und ähnliche Fragen wurden in unterschiedlicher Weise in den vorliegenden Beiträgen aufgegriffen.

Martin Nissen untersucht in seinem Beitrag Geschichtsschulbücher für Mädchen im 19. Jahrhundert und zeigt deren geschlechtsspezifische Besonderheiten auf. Geschichtsunterricht für Mädchen sollte statt der Vermittlung ereignisgeschichtlicher Fakten vor allem dazu dienen, christliche, später auch patriotische Moral zu vermitteln und den Schülerinnen ganz im Sinne einer historia magistra

11 Die Beiträge gehen auf eine von der DFG-Forschergruppe $»$ Historische Lebenswelten in populären Wissenskulturen der Gegenwart« im Dezember 2011 in Zusammenarbeit mit dem »Arbeitskreis Historische Frauen- und Geschlechterforschung (AKHFG)« veranstaltete Tagung zurück. Tagungsbericht Geschlecht und Geschichte in populären Medien. 01.12.2011-03.12.2011, Freiburg im Breisgau, in: H-Soz-u-Kult vom 16.02.2012 (http://hsozkult.geschichte.hu-berlin.de/tagungsberichte/id=4069). Zugriff am 26.4.2013. 
vitae historische Vorbilder zu präsentieren. Im höheren Mädchenschulwesen wurde Geschichte bevorzugt als Kultur- und Sozialgeschichte gelehrt, die Politikgeschichte rückte vergleichsweise in den Hintergrund, d.h. die (Schul)Geschichtsbücher des 19. Jahrhunderts waren per se und in ihren Inhalten gegendert. Die Darstellungen in den Schulbüchern wurden personalisiert und emotionalisiert. Diese Formen der Veranschaulichung rückten Mädchenschulbücher in die Nähe populärer Geschichtsdarstellungen ihrer Zeit.

Jasmin Meier und Anabelle Thurn untersuchen Geschichtsschulbücher des 21. Jahrhunderts unter der Fragestellung, wie diese Frauen- und Geschlechtergeschichte thematisieren. Die Darstellung antiker Geschlechterverhältnisse, so ihre zentrale These, die sie anhand eines deutsch-türkischen Vergleichs aufstellen, applizieren moderne Geschlechterverhältnisse auf die Antike. Dies zeigt sich nicht nur in den Texten, sondern insbesondere in den Deutungen bildlicher Darstellungen. Sie spiegeln zudem die national unterschiedlichen Historiographietraditionen, so z.B. im türkischen Fall einen geographisch-räumlichen Zugang zu Geschichte, im deutschen Fall das frühere Aufgreifen sozialgeschichtlicher Perspektiven. Der Vergleich zeigt, dass sich die Darstellung der antiken Geschlechterverhältnisse und -rollen entsprechend der national unterschiedlichen Geschlechterdiskurse teilweise signifikant unterscheidet. Gemein ist den neuesten Schulbuchproduktionen beider Länder jedoch die anachronistische Projektion, die Menschen der Antike trotz höchst unterschiedlichem sozialem und rechtlichem Status primär als »römische Hausfrauen und Geschäftsmänner« präsentiert.

Eine vielleicht noch breitere RezipientInnenschicht als das Schulbuch wies im 19. Jahrhundert das Medium der Zeitschriften, hier insbesondere der Familienzeitschriften, auf. In einem deutsch-britischen Vergleich untersuchen zwei Beiträge den Stellenwert von Frauen- und Geschlechtergeschichte in Zeitschriften des 19. Jahrhunderts. Beide Beiträge zeigen, dass sich bereits im 19. Jahrhundert in der populären Geschichtsschreibung Traditionslinien einer gegenderten, auf unterschiedliche geschlechtsspezifische Publika abzielenden populären Geschichtsschreibung ausbildeten, die sich nach Inhalt und Darstellungsform deutlich von akademischen Geschichtspräsentationen unterschied.

Doris Lechner und Nina Reusch machen in ihrer Untersuchung deutscher und britischer Familienzeitschriften die Zusammenhänge von Geschlechter- und Kulturgeschichte deutlich und überprüfen die These eines intersektionalen $\mathrm{Zu}$ sammenhangs von Gender und Genre. Familienzeitschriften, so zeigen sie am Beispiel von Modegeschichte auf, schrieben Geschichte für ein geschlechtlich nicht spezifisches Publikum, doch bot das Genre einen doppelten Raum für Frauen in der Geschichte: als Autorinnen wie als historische Akteurinnen. 
11

Barbara Korte und Sylvia Paletschek richten ihren Fokus auf feministische Zeitschriften und untersuchen, wie die bürgerlichen Frauenbewegungen beider Länder den Rückgriff auf Freiheits- und Emanzipationsbewegungen, auf >große Frauen< und auch >große Männer< der Geschichte zur Legitimation ihrer Emanzipationsforderungen nutzten. Frauengeschichte erlangte einen zentralen Stellenwert als Beleg für die intellektuelle, politische und ökonomische Leistungsfähigkeit von Frauen. Anfänge der Selbsthistorisierung zeigen sich aber auch, indem seit dem späten 19. Jahrhundert die >eigene< Geschichte, d.h. die Geschichte der Frauenbewegungen, aufgearbeitet wurde. Ansatzweise fanden in den Zeitschriften der Frauenbewegung auch Metareflexionen über das Schreiben von Geschichte statt. Der >allgemeinen< männlich konnotierten Geschichte wurde hier eine weibliche, sehr stark intentional ausgerichtete Gegengeschichte gegenübergestellt.

In unterschiedlichen Printmedien vom späten 18. Jahrhundert bis in die Gegenwart untersucht Sylvia Schraut fiktionale und faktuale Darstellungen der gewalttätigen Attentate von Charlotte Corday (1768-1793), die 1793 den Revolutionär Jean Paul Marat erstach, sowie von Karl Ludwig Sand (1795-1820), einem Burschenschaftler, der 1818 August von Kotzebue, einen vermeintlichen Spion der Reaktion, erdolchte. Beide sind bis heute zentrale Figuren in der Auseinandersetzung mit Terrorismus und der Konstruktion einer vom 19. Jahrhundert bis in die Gegenwart reichenden terroristischen Traditionslinie, in der Geschlecht eine zentrale Rolle zukommt. Die Auseinandersetzung mit den Gewalttaten Cordays und Sands wurde und wird vom 19. bis ins 21. Jahrhundert immer als Auseinandersetzung mit deren Geschlecht und als Infragestellung ihrer geschlechtlichen Identität geführt. Während in der Rezeption der Tat von Corday verschiedene Strategien gefunden werden mussten, Cordays geschlechtliche Identität ihrer als unweiblich markierten Tat anzupassen, war die SandRezeption durch ein changierendes Verhältnis von Männlichkeits- und Weiblichkeitszuschreibungen geprägt, das auch die politische Haltung der RezipientInnen spiegelte; so wurde und wird die Anwendung von Gewalt statt politischer Rationalität z.B. von Sands Kritikern weiblich gelesen.

Auf einen Kriminalfall des 19. Jahrhunderts bezieht sich der Geschichtscomic »Gift« (2010), den Sylvia Kesper-Biermann in ihrem Beitrag analysiert. Sie zeigt die Verschiebung der Handlungsräume historisch-fiktionaler Stoffe durch die Einführung weiblicher Hauptfiguren und durch das Aufkommen neuer Genres und sie diskutiert das Verhältnis von akademischer Geschichtsschreibung und populären Bezügen im Comic. »Gift« stellt weibliche Handlungsspielräume und Geschlechterverhältnisse des 19. Jahrhunderts in biographischer Perspektive aus einem weiblichen Blickwinkel dar. Der Geschichtscomic als eigenständiges 
populärhistorisches Genre ermöglicht nicht nur die Darstellung neuer Themen und Figuren, sondern auch ein Spiel mit fiktionalen Elementen und Authentizitätsverweisen. Populäre Geschichte und gerade neuere Genreentwicklungen im Comic, so die These, bergen die Möglichkeit, Geschlecht und historische Geschlechterverhältnisse mit einer erweiterten Perspektive darzustellen, die auch weibliche Räume und subjektive Perspektiven einschließt.

Dass ein weiblicher Blick auf historische AkteurInnen zu einer anderen Historiographie führen kann, zeigt Jule Nowoitnick in ihrem Beitrag über Tschingis Khaan-Romane der letzten zwei Dekaden, die von Frauen verfasst und tendenziell für ein weibliches Publikum geschrieben wurden. Khaan (um 1160-1227) wurde von männlichen Autoren stets als Herrscher und Kämpfer präsentiert; die Frauen in seinem Leben fanden kaum als eigenständige Akteurinnen Erwähnung. In den von Autorinnen geschriebenen historischen Romanen wird die maskulin-heterosexuelle Identität der männlichen Hauptfiguren durch homosexuelle Handlungen gebrochen. Mit einer Verschiebung der Handlungsräume vom Schlachtfeld auf den privaten Bereich des Alltags und der persönlichen Beziehungen erscheinen Frauen als handelnde Individuen. Diese Aufwertung weiblicher historischer Figuren geht einher mit Projektionen moderner Geschlechterverhältnisse in die Zeit des Mittelalters: Die Protagonistinnen vertreten eine moderne Ethik und erscheinen auf anachronistische Weise emanzipiert.

Mit Presseerzeugnissen, jetzt aber aus der Mitte des 20. Jahrhunderts, beschäftigt sich Leonie Treber, die am Beispiel der zeitgenössischen Darstellung von Trümmerfrauen die Wirkmächtigkeit populärer Medien, in ihrem Fall der Tagespresse, in der Konstruktion historischer Geschlechterbilder aufzeigt. Die Fïgur der Trümmerfrau entstand durch eine Pressekampagne innerhalb der SBZ. Sie sollte die Trümmerräumung von einer ungeliebten und als Strafarbeit konnotierten Arbeit zu einer ehrenvollen und für Frauen attraktiven Aufgabe machen. In der SBZ war die Trümmerfrau ein Symbol des Wiederaufbaus, während sie in den übrigen Besatzungszonen kaum, und wenn nur mit Bezug auf Berlin und die SBZ rezipiert wurde. Trümmerräumung wurde in der Nachkriegszeit meist von professionellen Unternehmen übernommen oder als Strafarbeit aufgefasst. Der Einsatz von Frauen war eher ein regionales, auf Berlin und die SBZ konzentriertes Phänomen. Dennoch erwies sich das zunächst durch die zeitgenössische Tagespresse geschaffene Geschichtsbild der Trümmerfrau über die Jahrzehnte und die Systemwechsel hinweg in unterschiedlicher Weise funktional und wandelbar und fand in einer mysthifizierten Form seit den 1980er Jahren breiten Eingang in die gesamtdeutsche populäre Geschichtskultur.

Ähnlich reflektiert Andre Dechert in seinem Beitrag, wie zeitgenössische, nun jedoch fiktionale Bilder in der populärhistorischen Rezeption mythisiert 
werden und so allmählich den Anschein historischer Fakten annehmen. In einer Rezeptionsanalyse US-amerikanischer Familiy Sitcoms der 1950er und 1960er Jahre, die er als Teil eines breiteren Geschlechter- und Familiendiskurses begreift, zeigt er, wie die in den Sitcoms konstruierten traditionalen Modelle der bürgerlichen Kernfamilie im Laufe der Rezeptionsgeschichte verschieden interpretiert und bewertet wurden. Parallel zum Wandel gesellschaftlicher Wertvorstellungen und Geschlechterdiskurse bewegte sich die Rezeption der Serien zwischen Idealisierung und Ablehnung des bürgerlichen Familienideals. Gleichzeitig verlief die Diskussion im Spannungsverhältnis von Fiktion und historischer Realität - während eine Lesart die Serien als fiktionale und idealisierende Produkte wahrnahm, erhielten sie in einer anderen als vermeintlichem Abbild historischer Realität Quellencharakter.

Traditionale Geschlechterverhältnisse der 1960er Jahre strukturieren auch die US-Serie »Mad Men«, die Renée Winter in ihrem Beitrag untersucht. Mit den Begriffen Wiederholung, Fortschritt und Rekonstruktion entwickelt sie ein methodisches Instrumentarium zur Analyse von Geschlechterdarstellungen in populären Geschichtsformaten. Das Konzept der Wiederholung macht sichtbar, wie Sexismus, aber auch Rassismus und Homophobie nicht allein dargestellt, sondern durch Kameraführung und Marginalisierung diskriminierter Figuren reinszeniert und somit wiederholt werden. Mit dem Begriff Fortschritt reflektiert Winter das teleologische Narrativ der Serie, die nicht nur die Emanzipation ihrer Hauptdarstellerin darstellt, sondern auch impliziert, in der gegenwärtigen Gesellschaft sei - im Gegensatz zu der der 1960er Jahren - das Ziel der Gleichberechtigung erreicht. Gleichzeitig erzählt die Serie aber auch vom technischen Fortschritt, der vor allem anhand des Mediums Fernsehen inszeniert wird. Rekonstruktion bezeichnet die Verhandlung weißer heterosexueller Männlichkeiten, die in »Mad Men« im Mittelpunkt steht. Die Serie zeigt, welchen Aufwand die Herstellung dieser Männlichkeiten bedeutet und wie neue männliche Identitäten, die sich den Anforderungen der Emanzipation anpassen müssen, formuliert werden.

Während die beiden Beiträge über Serien Zusammenhänge zwischen Geschlechterdarstellungen und Heteronormativität reflektieren, zeigen Beispiele aus der russischen sowie litauischen populären Geschichtskultur die enge Verbindung von Geschlecht und nationaler Identität auf. So legt Regine Nohejl am Beispiel verschiedener Werbespots der russischen Bank Imperial und des Spielfilms Napoleon kaput - Rževskij protiv Napoleona (Napoleon kaputt - Rževskij gegen Napoleon, 2012) dar, wie das Napoleon-Narrativ für den Diskurs nationaler Identität des gegenwärtigen Russland genutzt wird. Russland wird in diesen populärhistorischen Produktionen mit männlichen wie weiblichen Attributen 
1

versehen und kann gerade durch die Vereinigung beider Geschlechter und ihrer Tugenden zurn russisch-christlichen »All-Menschen« dem Eroberer Napoleon trotzen - ein Motiv, dass in beiden Beispielen jedoch nicht nur reproduziert, sondern auch ironisiert wird.

Gintare Malinauskaite untersucht die Erinnerungskultur Litauens, die durch eine zweifache Erinnerungskonkurrenz charakterisiert ist: In Konkurrenzverhältnissen stehen einerseits Gedenken an die NS-Zeit und an die Sowjet-Besatzung, andererseits die Erinnerung an den Holocaust und an den litauischen Partisanenkampf gegen Nazis wie Sowjets, wobei letzterer die litauische Erinnerungskultur und Identität dominiert. Der Holocaust, lange ein Tabuthema der litauischen Geschichtskultur, wird erst seit einigen Jahren verstärkt thematisiert. Das Beispiel des deutsch-litauischen Films »Ghetto (2006) zeigt, wie die populärkulturelle Erinnerung an den Holocaust mit einer viktimisierenden und objektivierenden Darstellung vor allem weiblicher Opfer zusammengeht. Der Film bedient sich eines voyeuristischen Blicks, der sexualisierte Gewalt an jüdischen Frauen zeigt und reproduziert, ohne sie als solche zu benennen - die Gewalt wird stattdessen als Liebesgeschichte inszeniert. Eine Möglichkeit der Selbstermächtigung jüdischer Frauen zu Subjekten und »Regisseurinnen der Erinnerung« bieten hingegen Dokumentationen, die ehemalige Partisaninnen zu Wort kommen lassen und nicht nur ein Gegennarrativ zu bisherigen Holocaust- oder Widerstandserzählungen bieten, sondern beide Aspekte der Erinnerungskultur zusammenbringen.

Zwei Beiträge reflektieren schließlich, wie populäre Geschichte Ausgangspunkt für doing gender sein kann. Am Beispiel eines Biedermeiermarkts analysiert Michaela Fenske das Spannungsverhältnis von gegenwärtigen und historisčthen Geschlechterrollen. Die Teilnehmer und Teilnehmerinnen des Marktes schlüpfen vor allem durch Kostümierung in historische Rollen, in denen sie auch Geschlechtererfahrungen machen, die quer zu ihren Rollen im alltäglichen Leben liegen. Zeitreisen, so die These, sind dabei keineswegs eine Rückkehr in konservative Geschlechterrollen, sondern vielmehr eine Alteritätserfahrung für Teilnehmende, die durch performativ-spielerische Reinszenierung traditioneller Weiblichkeiten und Männlichkeiten im Raum des Biedermeiermarkts Erfahrungen machen können, die ihnen sonst nicht möglich sind.

Geschlechterperformance mithilfe historischer Geschlechterrollen kennzeichnet auch die Szene des skandinavischen Viking Metal, wie Imke von Helden in ihrer Untersuchung textueller und visueller Geschlechtersymbolik von Metalbands zeigt. Die heteronormativ geprägte Metalszene vollzieht im Subgenre des Viking Metal noch eine weitere Aufwertung martialischer Männlichkeiten. Mit der Figur des Wikingers wird vor allem Krieg und Schlacht verbunden, was in Songtexten wie performativ auf der Bühne und in Musikvideos dargestellt 
11

wird. Diese männlichen Schlachtenmythen werden allerdings teilweise gebrochen - durch Ironisierung oder durch die Aneignung der männlich konnotierten Technik des Growlings durch Sängerinnen; hier liegt ein Potential, die männliche Markierung des Genres aufzubrechen.

Deutlich wird in allen Beiträgen, dass erstens historische Darstellungen von Geschlecht immer Auseinandersetzungen mit gegenwärtigen Geschlechterverhältnissen und -diskursen sind. Populäre Geschichte ist häufig normativ und intentional; eigene gesellschaftliche Verhältnisse und Wertvorstellungen werden in die Vergangenheit projiziert. Dies wird besonders deutlich am Beispiel von Geschlecht, das in den populären Geschichtspräsentationen meist in ahistorischer Weise verhandelt wird, die dem mainstream des jeweiligen zeitgenössischen Geschlechterdiskurses entsprechen. Während bis in die 1960er Jahre hinein in der populären Geschichte vor allem konservative Geschlechterverhältnisse und bürgerliche Familienmodelle propagiert wurden, zeigt sich die Intentionalität und der ahistorische Umgang mit Geschlecht heute in den vielen emanzipierten Heldinnen vor allem fiktionaler Erzählungen, die letztlich Projektionen gegenwärtiger emanzipierter Geschlechterideale auf die Geschichte sind. Der Gegenwartsbezug offenbart dabei auch, dass Geschlecht eine Ressource des Anschlusses von Geschichte an die Lebenswelt der RezipientInnen ist. Geschichte braucht Anknüpfungspunkte an die Erfahrungen des Publikums populärhistorischer Formate, um verstehbar und rezipierbar zu sein. Darstellungen von Geschlecht und Geschlechterrollen öffnen Erfahrungsräume, in denen sich alle RezipientInnen auskennen - so bestehen Möglichkeiten des direkten Vergleichs mit der eigenen Lebenswelt, des Wiederfindens eigener Erfahrungen in der Geschichte oder der Erfahrung von Alterität.

Zweitens steht die Frauen- und Geschlechtergeschichte in populären Genres der Kultur- und Alltagsgeschichte nah. Populäre Geschichte, die eine Plattform für beide Ansätze ist, eröffnet nicht nur eine Perspektive auf Frauen als Akteurinnen der Geschichte, sondern weitet auch den Horizont historischer Untersuchungsgegenstände hin zu Alltag und Familienleben, zu Emotionen und subjektiven Erfahrungen. Dass in den Beiträgen des Bandes häufiger Frauengeschichte und andere >weiblich $<$ markierte populäre Geschichtsdarstellungen untersucht wurden, liegt vermutlich daran, dass >männlichく markierte Bereiche populärer Geschichte nach wie vor ohne das Nachdenken über und Thematisieren von Geschlecht auskommen. Gleichzeitig scheint aber auch die geschlechtersensible Populärgeschichtsforschung vor allem auf Produkte, die Frauengeschichte verhandeln, zu fokussieren. 
Drittens ist der Umgang mit historischen Geschlechterverhältnissen in populären Geschichtskulturen national geprägt und gleichzeitig Nationsgrenzen transzendierend. Geschlecht hat eine wichtige Funktion für die Konstruktion nationaler Identitäten und Erinnerungskulturen. Doch neben nationalen Bezügen werden historische Geschlechterverhältnisse auch auf anderen Ebenen - lokalen, regionalen, transnationalen oder gar globalen - verhandelt. Wenn auch verstärkt sichtbar in den populären Geschichtsprodukten des 21. Jahrhunderts, so ist doch bereits im >nationalen<19. Jahrhundert eine Transzendierung des Nationalen zu beobachten, wenn etwa die jeweiligen nationalen Frauenbewegungen Frauen und Männer anderer nationaler Herkunft in ihre Ahnenreihe einspeisen oder wenn beispielsweise historische Gestalten wie die Französin Charlotte Corday oder der Deutsche Ludwig Sand zu Ikonen internationaler Diskurse über Terrorismus werden.

Dies führt zum vierten Punkt: Die hier vorgestellten Beiträge untersuchten in der Regel marktorientierte, auf ein westliches Publikum zugeschnittene Produkte. Die Themen, die populärhistorisch aufgegriffen werden, sind dabei teilweise, aber nicht zwingend an geschlechtsspezifische Publika geknüpft. Frauen waren seit dem 19. Jahrhundert wichtige Adressatinnen vor allem für fiktionale historische Stoffe, während der historische Sachbuchmarkt männlich markiert war. Die Grauzone zwischen den tendenziell für Männer bzw. für Frauen hergestellten Produkten populärer Geschichte, die auch schon im 19. Jahrhundert bestand, wird allerdings im 20. und 21. Jahrhundert immer größer.

Fünftens wird Geschlecht in populärhistorischen Diskursen vorwiegend innerhalb zweigeschlechtlicher Konzepte verhandelt. Alternative Formen von Geschlechtlichkeit innerhalb der populären Geschichte sind ein relativ junges Phänomen und finden, unabhängig vom Genre, nur wenig Platz. So sind zum Beispiel Elemente des Crossdressings oftmals eher einer bestimmten komödiantischen und damit genrespezifischen Tradition geschuldet, als dass sie subversive Möglichkeiten darstellen, Geschlechterrollen zu durchbrechen. Die performativen Formen populärer Geschichte bieten die Möglichkeit, mit Geschlechterrollen zu spielen und über das Eintauchen in Geschichte auch im Bereich des Geschlechts Alteritätserfahrungen zu machen. Doch diese Erfahrungen sind eher als unterhaltsames Spiel zu lesen, denn als Untergrabung von Geschlechterverhältnissen und -rollen. Sehr viel häufiger werden bestehende Geschlechterkonstellationen wiederholt und rekonfiguiert. Eine ambivalente oder nicht eindeutige Geschlechtsidentität dient meist der Abwertung der Person.

Sechstens: Zwar wandeln sich die in der populären Geschichtskultur vermittelten Wertvorstellungen mit der jeweiligen Gesellschaft und Zeit, doch zeigen sich gleichzeitig starke Pfadabhängigkeiten und damit Kontinuitäten vom 19. bis 
1. 1

ins 21. Jahrhundert. So wird Geschlecht meist nur dort als relevanter Faktor der Geschichte thematisiert, wo es um Frauen und weibliche Handlungsräume geht. Männlich markierte Bereiche gelten auch in der Populärgeschichte als >allgemeinhistorisch< und werden nicht mit Geschlecht in Verbindung gebracht. Die Beiträge dieses Bandes zeigen aber auch, dass populäre Geschichtsdarstellungen bisher schon wichtige Ansätze geboten haben, Frauen zu Subjekten wie Akteurinnen der Geschichtsschreibung zu machen. Sie haben das Potential, Geschlechterverhältnisse wie auch allgemeine Konzepte von Geschlecht in ihren historischen und geschichtskulturellen Dimensionen zu reflektieren. Aus Geschlechterperspektive sind populäre Geschichtsdarstellungen verglichen mit den akademischen nicht die >besseren< oder >schlechteren<, sondern attraktive und interessante, spielerisch wie normativ daherkommende >andere $<$. 


\section{1}

\section{LITERATUR}

Assmann, Jan (1988): »Kollektives Gedächtnis und kulturelle Identität«. In: Ders. (Hg.), Kultur und Gedächtnis, Frankfurt/Main: Suhrkamp.

Assmann, Jan (1992): Das kulturelle Gedächtnis: Schrift Erinnerung und politische Identität in frühen Hochkulturen, München: Beck.

Assmann, Aleida/Jan Assmann (1994): »Das Gestern im Heute: Medien und soziales Gedächtnis«. In: Klaus Merten/Siegfried J. Schmidt/Siegfried Weischenberg (Hg.), Die Wirklichkeit der Medien: Eine Einführung in die Kommunikationswissenschaft, Opladen: Westdt. Verl., S. 114-140.

Bennett, Judith M. (2006): History Matters: Patriarchy and the Challenge of Feminism, Philadelphia: PENN University of Pennsylvania Press.

Berger, Stefan (Hg.) (2008): The Contested Nation: Ethnicity, Class, Religion and Gender in National Histories, Basingstoke: Palgrave Macmillan.

Blos, Anna (1928): Frauen der deutschen Revolution 1848: 10 Lebensbilder $u$. e. Vorwort, Dresden: Kaden.

Bock, Gisela (1988): »Geschichte, Frauengeschichte, Geschlechtergeschichte «. * Geschichte und Gesellschaft 14.3, S. 364-391.

Bösch, Frank/Constantin Goschler (Hg.) (2009): Public History: Öffentliche Darstellungen des Nationalsozialismus jenseits der Geschichtswissenschaft, Frankfurt/Main: Campus.

Bruns, Claudia (Hg.) (2012): >Welchen der Steine du hebst<: Filmische Erinnerung an den Holocaust, Berlin: Bertz + Fischer.

Bruyn, Günther de (2002): »Königin Luise«. In: Etienne François/Hagen Schulze (Hg.), Deutsche Erinnerungsorte, München: C. H. Beck, S. 286-298.

Burstein, Miriam Elizabeth (2004): Narrating Women's History in Britain, 1770-1902, Aldershot: Ashgate.

Cornelißen, Christoph (2003): »Was heißt Erinnerungskultur? Begriff - Methoden - Perspektiven«. Geschichte in Wissenschaft und Unterricht 54, S. 548-563.

Cowman, Krista (2009): » There is so much, and it will all be History<: Feminist Activists as Historians, the Case of British Suffrage Historiography, 19082007«. In: Angelika Epple/Angelika Schaser (Hg.), Gendering Historiography: Beyond National Canons, Frankfurt/Main: Campus, S. 141-162.

Crane, Susan A. (2000): Collecting and Historical Consciousness in early Nineteenth Century Germany, Ithaca, NY: Cornell University Press.

Crivellari, Fabio et al. (Hg.) (2004): Die Medien der Geschichte: Historizität und Medialität in interdisziplinärer Perspektive, Konstanz: UVK. 
Epple, Angelika (2003): Empfindsame Geschichtsschreibung: Eine Geschlechtergeschichte der Historiographie zwischen Aufklärung und Historismus, Köln: Böhlau.

Epple, Angelika/Angelika Schaser (Hg.) (2009): Gendering Historiography: Beyond National Canons, Frankfurt/Main: Campus.

Epple, Angelika (2011): »Questioning the Canon: Popular Historiography by Women in Britain and Germany (1750-1850)«. In: Sylvia Paletschek (Hg.), Popular Historiographies in the 19th and 20th Centuries: Cultural Meanings, Social Practices, Oxford: Berghahn, S. 21-33.

Erll, Astrid (2005): Kollektives Gedächtnis und Erinnerungskulturen: Eine Einführung, Stuttgart: Metzler.

Eschebach, Insa (Hg.) (2002): Gedächtnis und Geschlecht: Deutungsmuster in Darstellungen des Nationalsozialistischen Genozids, Frankfurt/Main: Campus.

Falkenhausen, Susanne von (1997): »Geschichte als Metapher - Geschlecht als Symptom: Die Konstruktion der Nation im Bild«. In: Wolfgang Küttler/Jörn Rüsen/Ernst Schulin (Hg.), Geschichtsdiskurs. Band 3: Die Epoche der Historisierung, Frankfurt/Main: Fischer-Taschenbuch-Verl., S. 173-197.

Felber, Lynette (Hg.) (2007): Clio's Daughters: British Women Making History, 1790-1899, Newark/NJ: University of Delaware Press.

Fischer, Thomas/Rainer Wirtz (Hg.) (2008): Alles authentisch? Popularisierung der Geschichte im Fernsehen, Konstanz: UVK.

Förster, Birte (2004): »Das Leiden der Königin als Überwindung der Niederlage: Zur Darstellung von Flucht und Exil Luise von Preußens von 1870/71 bis $1933 \ll$. In: Horst Carl (Hg.), Kriegsniederlagen: Erfahrungen und Erinnerungen, Berlin: Akad.-Verl., S. 299-312.

Förster, Birte (2012): Popular History, Gender and Nationalism: Female Narratives of a National Myth«. In: Barbara Korte/Sylvia Paletschek (Hg.), Popular History Now and Then, Bielefeld: transcript, S. 149-168.

François, Etienne/Hagen Schulze (Hg.) (2002): Deutsche Erinnerungsorte, München: C. H. Beck.

Frevert, Ute (1988): »Bewegung und Disziplin in der Frauengeschichte - Ein Forschungsbericht«. Geschichte und Gesellschaft 14, S. 240-262.

Füßmann, Klaus/Heinrich Theodor Grütter/Jörn Rüsen (Hg.) (1994): Historische Faszination: Geschichtskultur heute, Köln: Böhlau.

Gehrke, Hans-Joachim/Miriam Sénécheau (Hg.) (2010): Geschichte, Archäologie, Öffentlichkeit: Für einen neuen Dialog zwischen Wissenschaft und Medien: Standpunkte aus Forschung und Praxis, Bielefeld: transcript. 
Glassberg, David (2001): Sense of History: The Place of the Past in American Life, Amherst: University of Massachusetts Press.

Grever, Maria (1997): »Die relative Geschichtslosigkeit der Frauen: Geschlecht und Geschichtswissenschaft«. In: Wolfgang Küttler/Jörn Rüsen/Ernst Schulin (Hg.), Geschichtsdiskurs. Band 4: Krisenbewusstsein, Katastrophenerfahrungen und Innovationen 1880-1945, Frankfurt/Main: Fischer-Taschenbuch-Verl, S. 108-123.

Grever, Maria (2008): Historical Culture, Plurality and the Nation-State: Lecture for the Forschungsgrippe Historische Lebenswelten. Albert-LudwigsUniversität Freiburg, 11.12.2008.

Grever, Maria (2009): »Fear of Plurality: Historical Culture and Historiographical Canonization in Western Europe«. In: Angelika Epple/Angelika Schaser (Hg.), Gendering Historiography: Beyond National Canons, Frankfurt/Main: Campus, S. 45-62.

Groebner, Valentin (2008): Das Mittelalter hört nicht auf: Über historisches Erzählen, München: Beck.

Groot, Jerome de (2009): Consuming History: Historians and Heritage in Contemporary Popular Culture, London: Routledge.

Hagemann, Karen/Jean H. Quataert (Hg.) (2008): Geschichte und Geschlechter: Revisionen der neueren deutschen Geschichte, Frankfurt/Main: Campus.

Halbwachs, Maurice (1985): Das kollektive Gedächtnis, Frankfurt/Main: Fischer-Taschenbuch-Verl.

Hardtwig, Wolfgang/Erhard Schütz/Ernst Wolfgang Becker (Hg.) (2005): Geschichte für Leser: Populäre Geschichtsschreibung in Deutschland im 20. Jahrhundert, Stuttgart: Steiner.

Hardtwig, Wolfgang/Alexander Schug (Hg.) (2009): History Sells! Angewandte Geschichte als Wissenschaft und Markt, Stuttgart: Steiner.

Hausen, Karin (1998): »Die Nicht-Einheit der Geschichte als historiographische Herausforderung: Zur historischen Relevanz und Anstößigkeit der Geschlechtergeschichte «. In: Hans Medick/Ann-Charlott Trepp (Hg.), Geschlechtergeschichte und allgemeine Geschichte: Herausforderungen und Perspektiven, Göttingen: Wallstein, S.15-55.

Heinze, Carl (2012): Mittelalter Computer Spiele: Zur Darstellung und Modellierung von Geschichte im populären Computerspiel, Bielefeld: transcript.

Horn, Sabine (2009): Erinnerungsbilder: Auschwitz-Prozess und MajdanekProzess im westdeutschen Fernsehen, Essen: Klartext Verlag.

Howsam, Leslie (2004): »Academic Discipline or Literary Genre? The Establishment of Boundaries in Historical Writing «. Victorian Literature and Culture 32.2, S. 525-545. 
Howsam, Leslie (2009): Past into Print: The Publishing of History in Britain 1850-1950, London: The British Library [u.a.].

Hunt, Lynn (1998): »The Challenge of Gender: Deconstruction of Categories and Reconstruction of Narratives in Gender History«. In: Hans Medick/AnnCharlott Trepp (Hg.), Geschlechtergeschichte und allgemeine Geschichte: Herausforderungen und Perspektiven, Göttingen: Wallstein.

Keilbach, Judith (2008): Geschichtsbilder und Zeitzeugen: Zur Darstellung des Nationalsozialismus im bundesdeutschen Fernsehen, Münster: LIT Verl.

Kircher, Marco (2012): Wa(h)re Archäologie: Die Medialisierung archäologischen Wissens im Spannungsfeld von Wissenschaft und Öffentlichkeit, Bielefeld: transcript.

Klein, Christa (2010): »Reflexive Authentizitätsfiktionen als situierte Geschichtsversionen am Beispiel des Living-History-Formats Die Bräuteschule 1958«. In: Ulrike Pirker/Mark Rüdiger et al. (Hg.), Echte Geschichte: Authentizitätsfiktionen in populären Geschichtskulturen, Bielefeld: transcript, S. 123-146.

Korte, Barbara/Ulrike Pirker (2011): Black History - White History: Britain's Historical Programme between Windrush and Wilberforce, Bielefeld: transcript.

Korte, Barbara/Sylvia Paletschek (Hg.) (2009a): History goes Pop: Zur Repräsentation von Geschichte in populären Medien und Genres, Bielefeld: transcript.

Korte, Barbara/Sylvia Paletschek (2009b): »Geschichte in populären Medien und Genres: Vom historischen Roman zum Computerspiel«. In: Dies. (Hg.), History goes Pop: Zur Repräsentation von Geschichte in populären Medien und Genres, Bielefeld: transcript, S. 9-60.

Korte, Barbara/Sylvia Paletschek (Hg.) (2012): Popular History Now and Then: International Perspectives, Bielefeld: transcript.

Langewiesche, Dieter (2008): Zeitwende: Geschichtsdenken heute, Göttingen: Vandenhoeck \& Ruprecht.

Lersch, Edgar/Reinhold Viehoff (2007): Geschichte im Fernsehen: Eine Untersuchung zur Entwicklung des Genres und der Gattungsästhetik geschichtlicher Darstellungen im Fernsehen 1995 bis 2003, Berlin: Vistas Verl.

Maitzen, Rohan Amanda (1998): Gender, Genre, and Victorian Historical Writing, New York: Garland.

Malečkova, Jitka (2004): »The Emancipation of Women for the Benefit of the Nation: The Czech Women's Movement«, in: Sylvia Paletschek/Bianka Pietrow-Ennker (Hg.), Women's Emancipation Movements in Europe in the 
19th Century: A European Perspective, Stanford: Stanford University Press, S. 189-205.

Maurer, Susanne (1996): Zwischen Zuschreibung und Selbstgestaltung: Feministische Identitätspolitiken im Kräftefeld von Kritik, Norm und Utopie, Tübingen: Ed. diskord.

Medick, Hans/Anne-Charlott Trepp (Hg.) (1998): Geschlechtergeschichte und allgemeine Geschichte: Herausforderungen und Perspektiven, Göttingen: Wallstein-Verl.

Meiners, Christoph (1788-1800): History of the Female Sex: Comprising a View of the Habits, Manners, and Influence of Women, Among All Nations, from the Earliest Ages to the Present Time, London: Henry Colburn.

Melman, Billie (2006): The Culture of History: English Uses of the Past 18001953, Oxford: Oxford University Press.

Mommertz, Monika (2010): »Geschlecht als Markierung, Ressource und Tracer: Neue Nützlichkeiten einer Kategorie am Beispiel der Wissenschaftsgeschichte der Frühen Neuzeit«. In: Christine Roll (Hg.), Grenzen und Grenzüberschreitungen: Bilanz und Perspektiven der Frühneuzeitforschung, Köln: Böhlau, S. 573-592.

Nissen, Martin (2009): Populäre Geschichtsschreibung: Historiker, Verleger und die deutsche Öffentlichkeit (1848-1900), Köln: Böhlau.

O'Dowd, Mary (Hg.) (2004): »History Women«. Storia della Storiografia 46 [special issue].

Offen, Karen (2000): European Feminisms, 1700-1950: A Political History, Stanford, Calif: Stanford University Press.

Opitz, Claudia (2010): Geschlechtergeschichte, Frankfurt/Main: Campus.

Oswalt, Vadim/Hans-Jürgen Pandel (Hg.) (2009): Geschichtskultur: Die Anwesenheit von Vergangenheit in der Gegenwart, Schwalbach/Ts: Wochenschau-Verl.

Paletschek, Sylvia/Bianka Pietrow-Ennker (Hg.) (2004): Women's Emancipation Movements in the Nineteenth Century: A European Perspective, Stanford: Stanford University Press.

Paletschek, Sylvia (2006): »Ermentrude und ihre Schwestern: Die ersten habilitierten Historikerinnen in Deutschland «. In: Henning Albrecht/Barbara Vogel (Hg.), Politische Gesellschaftsgeschichte im 19. und 20. Jahrhundert: Festgabe für Barbara Vogel, Hamburg: Krämer, S. 175-187.

Paletschek, Sylvia (2007): »Historiographie und Geschlecht«. In: Johanna R. Regnath/Mascha Riepl-Schmidt/Ute Scherb (Hg.), Eroberung der Geschichte: Frauen und Tradition, Hamburg: LIT-Verl., S. 105-127. 
Paletschek, Sylvia/Sylvia Schraut (Hg.) (2008a): The Gender of Memory: Cultures of Remembrance in Nineteenth- and Twentieth-Century Europe, Frankfurt/Main: Campus.

Paletschek, Sylvia/Sylvia Schraut (2008b): »Introduction: Gender and Memory Culture in Europe - Female Representations in Historical Perspective«. In: Dies. (Hg.), The Gender of Memory: Cultures of Remembrance in Nineteenth- and Twentieth-Century Europe, Frankfurt/Main: Campus, S. 7-28.

Paletschek, Sylvia/Sylvia Schraut (2008c): »Remembrance and Gender: Making Gender Visible and Inscribing Women into Memory Culture«. In: Dies. (Hg.), The Gender of Memory: Cultures of Remembrance in Nineteenth- and Twentieth-Century Europe, Frankfurt/Main: Campus, S. 267-287.

Paletschek, Sylvia (2009): »Opening up Narrow Boundaries: Memory Culture, Historiography and Excluded Histories from a Gendered Perspective«. In: Angelika Epple/Angelika Schaser (Hg.), Gendering Historiography: Beyond National Canons, Frankfurt/Main: Campus, S. 163-177.

Paletschek, Sylvia (Hg.) (2011): Popular Historiographies in the 19th and 20th Centuries: Cultural Meanings, Social Practices, Oxford: Berghahn.

Pietrow-Ennker, Bianka (1999): Rußlands sneue Menschen $<$ Die Entwicklung der Frauenbewegung von den Anfängen bis zur Oktoberrevolution, Frankfurt/Main: Campus.

Pirker, Eva Ulrike/Mark Rüdiger et. al. (Hg.) (2010): Echte Geschichte: Authentizitätsfiktionen in populären Geschichtskulturen, Bielefeld: transcript.

Rausch, Helke (2008): »Marginal Figure in the Nation: Gendered National Memories in Late Nineteenth-Century Western European Metropoles«. In: Sylvia Paletschek/Sylvia Schraut (Hg.): The Gender of Memory: Cultures of Remembrance in Nineteenth- and Twentieth-Century Europe, Frankfurt/Main: Campus, S.31-60.

Rendall, Jane (2004): »Recovering Lost Political Cultures: British Feminisms, 1860-1900«. In: Sylvia Paletschek/Bianka Pietrow-Ennker (Hg.), Women's Emancipation Movements in Europe in the 19th Century: A European Perspective, Stanford: Stanford University Press, S. 33-52.

Riepl-Schmidt, Maja (Hg.) (1998): Frauen und Revolution: Strategien weiblicher Emanzipation 1789 bis 1848, Tübingen: Silberburg-Verl.

Rosenzweig, Roy (1998): The Presence of the Past: Popular Uses of History in American Life, New York, NY: Columbia University Press.

Rüsen, Jörn (1994): »Was ist Geschichtskultur? Überlegungen zu einer neuen Art, über Geschichte nachzudenken«. In: Klaus Füßmann/Heinrich Theodor Grütter/Jörn Rüsen (Hg.), Historische Faszination: Geschichtskultur heute, Köln: Böhlau, S. 3-26. 
1

Samida, Stefanie (Hg.) (2011): Inszenierte Wissenschaft: Zur Popularisierung von Wissen im 19. Jahrhundert, Bielefeld: transcript.

Sánchez Marcos, Fernando (2009): Historical Culture. In: Culturahistorica (http://www.cultura-historica.es/sanchez_marcos/historical\%20_culture.pdf). Zugriff am 04.04.2013.

Schaser, Angelika (2012): »Der große Krieg in Deutschland: Literarische Geschichtsschreibung als weibliche Geschichtswissenschaft? «. In: Gesa Dane/Barbara Hahn (Hg.), Denk- und Schreibweisen einer Intellektuellen im 20. Jahrhundert: Über Ricarda Huch, Göttingen: Wallstein.

Schatzberg, Karin (Hg.) (1985): Frauenarchive und Frauenbibliotheken, Göttingen: Edition Herodot.

Scherr, Johannes (1860): Geschichte der deutschen Frauen: In drei Bänden nach den Quellen, Leipzig: Wigand.

Schilling, René (1998): »Die soziale Konstruktion heroischer Männlichkeit im 19. Jahrhundert: Das Beispiel Theodor Körner«. In: Karen Hagemann (Hg.), Landsknechte, Soldatenfrauen und Nationalkrieger: Militär Krieg und Geschlechterordnung im historischen Wandel, Frankfurt/Main: Campus,. S. 121-144.

Schneider, Corinna (2006): »Die Anfänge des Frauenstudiums in Europa: Ein Blick über die Grenzen Württembergs«. In: 100 Jahre Frauenstudium (http://www.uni-tuebingen.de/frauen-studium). Zugriff am 15. April 2013.

Schönemann, Bernd (2002): »Geschichtskultur als Forschungskonzept der Geschichtsdidaktik«. Zeitschrift für Geschichtsdidaktik 1, S. 78-86.

Smith, Bonnie (1984): »The Contribution of Women to Modern Historiography in Great Britain, France, and the United States, 1750-1940«. American Historical Review 89.3, S. 709-732.

Smith, Bonnie G. (2001): The Gender of History: Men, Women and Historical Practice, Cambridge: Harvard University Press.

Smith, Bonnie (2009): »Gendering Historiography in the Global Age: A U.S. Perspective«. In: Angelika Epple/Angelika Schaser (Hg.), Gendering Historiography: Beyond National Canons, Frankfurt/Main: Campus.

Stollberg-Rillinger, Barbara (1996): »Väter der Frauengeschichte? Das Geschlecht als historiographische Kategorie im 18. und 19. Jahrhundert«. Historische Zeitschrift 262, S. 39-71. 